\section{L'IMPORTANCE DE LA COMMUNICATION ECRITE ET VERBALE}

\section{CHEZ L'INGENIEUR}

\section{SONDAGE D'OPINIONS}

Par: Claude Guernier, Ing., Gaston Pouliot, Ph.D. Rolland Viau, M.A. Educ. et Fred Bassal, ing.

\section{Les origines du sondage}

L'enseignement de 1 a langue maternelle a toujours constitué une préoccupation majeure des milieux de l'éducation; le manque de maitrise du françals dont falt preuve un grand nombre de diplômés qui débouchent sur le marché du travail n'est une nouvelle pour personne. Au Québec, la série d'articles de Lysiane Gagnon (La Presse, avril 75) (4) représente sans doute l'analyse la plus alarmante des dernières années. Les constatations de la journaliste corroboralent celles effectuées par les professeurs de l'Ecole Polytechnique, dont plusieurs avaient, de leur propre Initiative, introdutt quelques palliatifs. Cette prise de conscience s'opérait en même temps que la refonte du programme des études du premier cycle, refonte qui mobilisait toutes les énergies.

Le nouveau programme fut adopté en janvier 77. Cela permit, dès février 77 , de mettre sur pied un "groupe d'étude sur la qualité du français écrit à l'Ecole Polytechnique".

Après quelques réunions, ce comité ad hoc énonçatt les principes ou conclustons sulvantes:

- Tout étudiant entrant à Polytechnique devrait posséder une connaissance minimale du français écrit;
- Si toutefois cette connaissance était insuffisante, 1 'Ecole devrait offrir au candidat-ingénfeur des cours de rattrapage en français ne comportant pas de crédits de scolarité;

- Cette connaissance du frança1s écrit devrait évoluer, au cours du séjour de l'étudiant à Polytechnique, en une apt1tude à rédiger correctement des rapports techniques;

- L'exposé oral occupe aussi une place importante dans les rapports de l'ingénieur avec son milieu de travall.

Ce mode d'expression exige également de l'esprit de synthèse, de la clarté et de la préctston. Il faudra donc y faire appel comme soutien d'un programme d'amélioration du françats écrit;

- Ce genre de formation à la communication technique relève de 1 a responsabilité d'une école d'ingénteurs.

Bien que ces conclusions afent été amplement justifiées par les opintons recuel11ies à l'intérieur de l'Ecole Polytechnique, 11 fallatt vértfier sil ce besoin d'améliorer les aptitudes à l'expression tant écrite que verbale chez les jeunes ingénieurs était ressenti par l'employeur et, par la sulte, déterminer une ligne d'action. C'est alnsi qu'en octobre 78, germa l'idée d'un sondage. On conçut le questionnaire donné en annexe I de ce texte. Il s'agissat alors de garantir un nombre suffisant de réponses et en même temps assurer un recouvrement adéquat des besoins et opinions des divers genres d'entreprises. 
Des professeurs de chacun des départements de l'Ecole Polytechnique contactèrent donc des ingénieurs occupant des postes de commande dans l'industrie et les services publics pour sollicter leur participation à ce sondage. Dans la majorité des cas une collaboration Immédlate nous fut acquise. Certalns y allèrent même de nombreux commentalres, références et documents.

Noụs avons reçu une quarantalne de réponses, $91 \%$ du nombre d'envols. Dans certaines entreprises plusteurs personnes ont rempli le questionnaire ou nous ont envoyé des commentaires. Le tableau qui suit résume les résultats de ce sondage.

\section{Résultats de 1'enquête}

1) Avez-vous (ou avez-vous eu) souvent à lire des

rapports présentés par des Ingénieurs subalternes? Très souvent

Peu souvent ou à l'occasion

34

Réponses non-quantifiables

4

Tota1

2) A votre avis, quel pourcentage de son temps

l'ingénieur passe-t-il à rédiger des rapports?

31 réponses quantifiables

Moyenne des chiffres donnés dans

$\begin{array}{lr}\text { ces réponses } & 23 \% \\ \text { Extrêmes } & 3-60 \%\end{array}$

3) Dans quelle mesure la qualité de ses rapports affecte la promotion d'un ingénieur? Très importante

Importante

16

Peu importante

Réponses non-quantifiables

4) En gênéral, les jeunes ingénieurs diplômés de Polytechnique ou d'ailleurs rédigent-ils des rapports satisfatsants?

$\begin{array}{lr}\text { Satisfaisants } & 3 \\ \text { Plus ou motns satisfaisants } & 1 \\ \text { Peu satisfaisants } & 30 \\ \text { Peu satisfaisants et même trop souvent } & 1 \\ \text { mauvais } & 3 \\ \text { Mauvais } & 1 \\ \text { Cela varie avec les Individus } & 1\end{array}$

5) A votre avis où se situent les points fatbles dans les rapports fournis par de jeunes ingénieurs?

\begin{tabular}{ll}
$\begin{array}{l}\text { Faibles- Moyen Satis- Total } \\
\text { se marquée }\end{array}$ & falsant \\
\hline
\end{tabular}

a) Structure du rapport (loglque, 31 organisation)

b) Manque de clarté

30

c) Manque de concision

d) Choix de termes

e) Présentation

f) Qualité des figures, diagrammes, tableaux

g) Autres:

- Maturité 1ittéraire

- Manque d'imagination et d'audace 
- Qualité du français et/ou de 1 'anglats

- Etapes sautées

- Ignorance du destinata1re

- Documentation incomplète

- Renseignements superfius

6) A quoi attribuez-vous ces falblesses?

a) Connatssance insuffisante de la langue 25

b) Connaissances techniques insuffisantes 2

c) Ignorance des exigences propres à 1 a rédaction technique

d) Manque d'esprit de synthèse

e) Autres commentalies reçus:

- sévérité Insuffisante des correcteurs 2

- manque de cours de rédaction

- coordination Inexistante entre spécialistes et entre professeurs pour fixer des normes minimales

- trop grand nombre de rapports de faible qualité

- les ingénfeurs ne lisent pas assez

- motivation absente

- objectifs mal compris

- rapport non préparé en fonction de la personne à laquelle 11 est destiné 1

- manque d'autodiscipline

7) Croyez-vous que ce problème soit suffisamment sérleux pour justifier l'inclusion d'un cours de rédaction technique au programme d'étude de 1'Ecole Polytechnique?

oui: 42

Non: 1

1 Ce cours devrait être donné par:

- professeur de Poly seul

- Ingénieur de 1'industrie seul

- professionnel de la communication seul

- professeur de Poly plus Ingénteur de l'industrie

- professeur de Poly plus professionnel de la communication

- ingénieur de l'industrie plus professionnel de la communication

- professeur de Poly plus ingénteur de l'industrie plus professionnel de la communication

- professeur de français avec assistance ingénieur de l'industrie plus professionnel de $1 a$ communication

- Ingénieur doté d'une formation en communication

- aucune suggestion

6

) Etes-vous satisfalt de l'aptitude des jeunes ingénleurs à présenter oralement leurs ldếes et leurs travaux lors de discussions de groupe, d'ateliers, de congrès?

\begin{tabular}{lr} 
Oul & 18 \\
Partiellement & 11 \\
Non & 9 \\
Aucune opinion & 2 \\
\hline & 40
\end{tabular}

nalyse des résultats

a majorité des personnes qui nous a répondu (83\%) talt blen apte à se prononcer sur ce problème, ayant u l'occasion de 1 ire de nombreux rapports d'Ingéleurs comme en témoigne notre première question. Le ut de notre seconde question n'étalt évidemment pas e chiffrer de façon immuable le temps que l'ingénieur 
moyen consacre à la rédaction de rapports. I1 s'aglssalt plutôt de déterminer si c'était là une activité Importante de l'ingénieur, activité à laquelle 11 consacre d'allleurs un temps très variable selon sa discipline. Toutefols, comme le souligne si justement un de nos interlocuteurs occupant un haut poste de direction dans un ministère du Québec:

"Le temps passé à la rédaction de rapports ne nous semble pas important. Le fait que chaque ingénieur doive nécessalrement rédiger des rapports sur ses travaux m'apparaft plus significatif. En effet, c'est à partir de ses rapports que l'ingénieur est Jugè :

même si ses études techniques sont excellentes, s1 le rapport qu' 11 rédige est falble, c'est l'ensemble de son travail qui sera jugé comme faible."

D'autres, parm1 les personnages sollicités, nous ont également fourni des réponses semblables. C'est d'allleurs ce falt, l'influence qu'exerce la qualité de ses rapports sur son avenir, que notre troistème question se proposalt d'élucider. La réponse est éloquente, $92 \%$ des personnes interrogées pense que la qua11 té de ses rapports affecte de façon déterminante la promotion future d'un ingénieur.

L'Importance du sujet étant établie, 11 fallatt ensulte évaluer dans quelle mesure les jeunes ingénieurs satisfaisalent à cette exigence de la profession; or, $85 \%$ des réponses à 1 a question no 4 indiquent que les rapports des jeunes diplômés sont peu satisfaisants. De ces premières données, 1 'importance et le besotn pour un jeune ingénieur de bien rédiger un rapport technique ressortent donc de façon claire.
La question $n^{0} 5$ tentalt de circonscrire le problème: "quelles sont les principales falblesses relevées dans les rapports?" On a noté en particu1ier:

- 1a structure du rapport

- le manque de clarté

- le manque de concision

- 1a présentation

- le choix de termes

Une remarque faite par un "surveillant de recherche" d'une 1mportante industrie métallurgique souligne un corollatre troublant de ces falblesses:

"Few reports, however, were poorly organized, which reflected well the state of the student's mind".

Cette personne considérait par allleurs que, dans l'ensemble, les stagiaires ( 11 s'agtssatt surtout d'emplots d'été) remettaient des rapports bien écrits. Ce qui inquiète dans cette remarque c'est justement la relation établie entre un rapport mal structuré et un certain désordre intellectuel chez son auteur. D'après notre enquête, 1 e manque de structure des rapports de jeunes ingénieurs semble être la falblesse la plus marquée. Si le manque d'ordre dans les idées d'un individu l'empêche de rédiger un texte cohérent, 11 semble logique de conclure que les falblesses indiquées en deuxième et troisième positions, come le manque de clarté et de concision, découlent elles aussi de cette même cause: un premier pas vers la rédaction d'un texte clałr nécessite inévitablement une classification des idées et des données. 
Un ancien directeur de l'ordre des ingénieurs du Québec nous soulignalt justement, dans une conversation téléphonique, le fait qu'en rédigeant des rapports 1 'étudiant apprenait à mieux organiser ses idées. C'est là un argument de poids en faveur d'une plus grande vigilance de la part des professeurs en ce qui concerne les rapports d'étudiants.

Quant aux causes de ces falblesses, c'est bien le "manque de formation à la rédaction technique" (88\% des réponses) qui semble recuelilir le plus de suffrage, sulvi d'allleurs par la "connalssance Insuffisante de 1a langue" (61\%) et le "manque d'esprit de synthèse" (41\%). I1 est Intéressant de constater que la "falblesse des connaissances techniques" est à peine évoquée (5\%). En ce qui concerne le manque d'esprit de synthèse, le chiffre cité c1-haut peut être trompeur; car ce facteur n'a été suggêré que dans une version révisée du questionnatre envoyée après qu'un certałn nombre de réponses nous solt déjà parvenu. Si l'on ne considère que ce dernier groupe, le pourcentage monte à 72\%; cela ramène ce paramètre au rang des causes majeures. N'est-11 pas, 1 il aussi, un indice d'un manque d'ordre dans les idées?

Avant de clore cette analyse de la question no 6,11 convient d'évoquer une cause qui modifiera sans doute, quolque indirectement, ces premières conclusions. Des questions posées à des étudiants de différents départements de 1 'Ecole ont révélé que peu d'entre eux mesurent correctement 1 'importance des commnications, écrites ou verbales, dans leur future carrière. Notre groupe d'étude a déjà préconisé des mesures qui afderaient à sensibiliser l'étu- diant à l'importance de cette ativité (6).

Prévoyant une certalne insatisfaction dans le milieu du travall à l'égard de nos diplômés sous l'angle de la communication écrite, nous avons alors demandé, dans notre septième question, si 1 'Ecole Folytechnique devalt instituer un cours de rédaction technique. $98 \%$ des réponses furent affirmatives. Il ne faudrait, néanmolns, pas crolre qu'on attendalt tout de cette mesure, et bien des mises en garde nous ont été servies, comme celleci venant du président d'une importante société mintère:

"Je ne crois pas que la direction de l'Ecole obtiendra le résultat escompté par le seul falt d'organiser un tel cours. Le cours en sol ne peut que donner un aperçu de la structure générale d'un rapport et Informer quant aux sources de renseignements disponibles, quant aux vocabulaires techniques et usuels, quant aux modes et moyens de présentation,êt susciter un intérêt à la bonne communication des informations que l'ingénieur a acquises et qu'il désire transmettre.

L'aptitude à écrire de bons rapports est surtout affaire de pratique, d'expériénce et aussi de fierté dans son travail. C'est pourquoi, à mon avis, ce qui importe surtout c'est le nombre d'occasions que la direction et le collège des professeurs de l'Ecole donneront aux étudiants, durant leur séjour à 1'école, de préparer des rapports selon des normes établies. Je suppose évidemment que chacun des membres du corps professoral est conscient de sa responsabilité : 11 dolt non seulement s'assurer que l'élève acqulert 
les connalssances nécessalres, mais aussi qu' 11 devienne apte à bien les exprimer. En bref, tout rapport mérite qu'11 soit bien falt et doit être côté sur ce plan tout comme sur 1 e contenu technique".

En ce qui à trait à la responsabilité d'un tel cours (question no 8), les avis semblent très partagés quoique 1 'on puisse déceler une tendance en faveur des professionnels de la communication. Nous avons souligné au début de ce texte l'apport que peut constituer l'exposé oral comme moyen d'alder l'étudiant à structurer sa pensée, c'est pourquol les dernières questions de notre enquête portalent sur ce mode de communication. Les réponses expriment un peu plus de satisfaction en ce qui à tratt à la communication verbale qu'en ce qui concerne l'aptitude à écrire des jeunes ingénieurs. Toutefols, personnes souhatiatent qu'on fournisse aux êlèves-ingénteurs plus d'occastons de s'exprimer en public; 11 seratt bon d'utıliser plus fréquemment le mode d'enseignement par séminaires.

Enfin, nombreux sont ceux qui, à notre demande, nous ont falt d'utiles suggestions au sufet de la structure d'un cours de rédaction technique. Ces réponses constitueront une alde précleuse dans l'élaboration du programme de ce cours.

\section{Conclusion et recommandations}

Ce sondage révèle qu'un besoln existe et qu'i1 faut prendre des mesures pour corriger la situation.

Ce besoin n'est pas particulier au milieu québécols francophone. De semblables constatations ont été faltes à la sulte d'enquêtes réalisées aux Etats-Unis, auss 1 bien dans certaines univer- sités $(1,2,5$,$) que dans 1$ 'industrie $(3,5)$.

Plusieurs institutions canadiennes se sont aussi dotées de cours de rédaction de rapports ou de communication (cf. ref. 6, p. 15). Par ailleurs, 11 existe sur le marché un certain nombre d'organismes professionnels qui offrent des services de rédaction de rapports, rédaction de soumissions, etc... et, d'autres, des cours intensifs de communication pour ingénieurs $(7,8)$. Cela ne constitue-t-il pas autant dindices de besoins ressentis dans toute 1 'Amérique du Nord? Les étudiants qui entrent à Polytechnique n'ont certes pas reçu une préparation suffisante en français au secondaire et au CEGEP, mais l'Ecole ne peut rejeter pour autant la responsabilité qui lul incombe, celle d'apprendre aux élèvesingénieurs, malgré ce handicap, à rédiger de bons rapports techniques qui sauront satisfaire aux exigences de l'employeur et de 1 a professton.

Parmi les mesures à prendre, un cours de rédaction technique constitue l'élément central. Toutefols, son Influence peut s'avérer négligeable $s^{\prime} 11$ n'est pas accompagné d'une action concertée de tous les professeurs.

Au-delà du vocabulaire qui permet d'exprimer sa pensée de façon plus précise s'il est entendu, au-delà de la grammaire qui permet de construire des phrases claires et logiques, 11 existe l'art de présenter un rapport, un art qui s'apprend par 1a répétition et par la correction des erreurs. C'est là que tous les professeurs dolvent intervenir.

Comme bénéfices apparents immédiats, on peut espérer que les diplômés de Polytechnique sauront mieux se faire comprendre. De plus, si l'ordre d'un 
rapport refiète 1 'ordre dans les idées de son auteur, on peut espérer plus encore que le jeune ingénieur aura appris à mieux structurer sa pensée. Cela pourra aider non seulement dans la communication avec son milieu mals aussi dans 1 'analyse même des problèmes qu'11 devra résoudre.

On ne soulignera jamais assez l'importance que revêtent de bonnes communications dans l'efficaclté d'une entreprise. C'est ce sur quoi M. André Tasso voulait attirer l'attention du lecteur dans un article publié dans Plan, le journal de l'ordre des ingénteurs du Québec (9). La rédaction technique ne représente qu'un aspect de ce réseau de communications. Mats, qui pourra compter la somme d'énergie (et d'argent) Investie en pure perte dans des études approfondies, auxquelies on $n$ 'a donné aucune suite, tout simplement parce que le rapport dans lequel leurs résultats étalent consignés était mal présenté?

Le dialogue est ouvert: Si nos lecteurs désirent nous envoyer des commentaires ou remplir le questlonnalre, nous apprécierons beaucoup cette co1laboration de leur part. L'opinion de jeunes Ingénieurs nous seralt de la plus grande utilité; $11 s$ pourratent confirmer $s i$, pour eux, le problème possède bien l'acuité que nous lui accordons dans ces pages.

\section{Références}

1. HAND, H.E. (1964), "An attempt to Measure Success in Technical Writing", Jour. Eng. Educ. 55 , no 3, pp. $72-76$.
2. ESTRIN, H.A. (1968), "Teaching Report Writing", Civil Engineering E., Dec. 68, p. 65.

3. HENDERSON, B.C. (1973), "Employers speak Out on Employees", Geotimes, Oct. 1973, pp. 37-38.

4. Gagnon, Lysiane (1975), "Le drame de l'enseignement du français" articles publiés dans La Presse du 5 au 12 avr11 1975 .

5. ALEXANDER, J.A. (1977), "Communications Skil1sEaster Success and Academic Success", The Mines Mag., Oct. 1977, PP. 11-27.

6. Groupe d'étude sur la qualité du françals écrit à Polytechnique (1977), rapport no 2 (Programme d'implantation), Document CE 223, 19 déc. 1977.

7. Association of Professional Engineers of ontario (1978), APEO Seminar:"Writers" Workshop for Engineers", The Ontario Digest and Engineering, Digest, Août 1978, p. 21 .

8. Volr le contenu du cours intitulé "Writing Skilis for Sclentists and Engineers", American Institute for Professional Education Inc.

9. TASSo, André J. (1978), "Connalssez-vous 1a néguentropie?", Journal Plan, Août-septembre 1978, p. 3 . 
Les auteurs tiennent à remercler toutes les personnes qui ont accepté de répondre aux questions de ce sondage; l'espace dispontble dans cette revue ne leur permet toutefois pas d'en donner 1 a 11ste. Ils veulent aussi remercier leurs collègues du groupe d'étude sur la qualité du français et du sous-comité sur la rédaction technique dont la collaboration a permis la réalisation de cette enquête. I1 s'ag1t de MM. Chris BALE (Gếnie Métallurgique), Françols BRIERE (Génie Civil), Tuong BUI ThIEU (Génie Physique), Jean Corneille (Gếnie Chimlque), Eric DEVLIN (Association des Etudiants), Arnold DRAPEAU (Génie Civil), Gabriel Garneau (Mathématiques), Mile Cécile GHYS (Assoclation des Etudiants), Michel GoU (Génle Mécan1que), Jean Granger (Génte Civil), Dantel ROSON (Génie Nucléa1re), Jean Rousselle (Génie Civi1), Jacques SPOONER (Génie Electrique), tous de l'Ecole Polytechnique de Montréal.

Cet article a paru d'abord dans l'INGENIEUR, Janvier/février 1979. Le droit de réproduction en a été accordé par l'éditeur.
ENQUETE REDACTION TECHNIQUE ET

\section{COMMUNICATION VERBALE}

1) Avez-vous (ou avez-vous eu) souvent à l1re des rapports présentés par des ingénieurs subalternes?

Très souvent à 1 'occaston Peu souvent

2) A votre avis, quel pourcentage de son temps 1 'ingénieur passe-t-11 à rédiger des rapports?

3) Dans quelle mesure la qualité de ses rapports affecte la promotion d'un ingénteur?

Très Importante 1mportante Peu importante

4) En général, les jeunes ingénieurs diplômés de Polytechnique ou d'allieurs, anglophones ou francophones, rédigent-1ls des rapports satisfaisants? Satisfaisants peu satisfaisants mauvais

5) A votre avis quels sont les points falbles des rapports fournts par les jeunes ingénieurs?

Fafblesse Satisfaisant

structure du raparquée

port (logique,

organisation)

b) manque de clarté

c) manque de concision 
d) choix de termes

e) présentation

f) qualité des figures diagrammes, tableaux

g) autres

6) A quol attribuez-vous ces falblesses?

a) connatsance insuffisante de la langue

b) connaissances techniques insuffisantes

c) ignorance des exigences propres à la rédaction technique

d) manque d'esprit de synthèse

e) autres

7) Croyez-vous que ce problème de rêdaction technique soit suffisamment sérieux pour justifier l'inclusion d'un cours de rédaction technique au programme d'études de 1 'Ecole Polytechnique?

ou 1

Non

8) Si tel est le cas,

a) ce cours devra1t-11 être donné:

- par des professeurs de Polytechnique

- par des ingénieurs travaillant dans I'industrie, les services publics, etc...

- par des professionnels de la communication

- commentaires:

b) pouvez-vous offrir des suggestions en ce qui concerne le contenu de ce cours? ou 1

Non

(S.V.P. les donner sur une feut11e séparée)

9) Etes-vous satisfait de 1 'aptitude des jeunes ingénleurs à présenter oralement leurs ldées et leurs travaux lors de discussions de groupe, d'ateliers, de congrès?

Oui Partiellement Non

10) Quelies mesures devrait-on adopter pour améllorer le mode d'expression verbal chez les jeunes Ingénteurs?

NOTE: Nous apprécierons tout commentaire supplémentaire que vous jugeriez utile d'ajouter à ce questionnaire que vous voudrez bien adresser à M. Claude Guernter, ing. Ecole Polytechnique, case postale 6079, Succursale A, Montréal H3C 3A7.

\section{Les auteurs:}

M. CLAUDE GUERNIER, ing., est professeur agrégé au Département de Génie Mécanique de l'Ecole Polytechnique de Montréal. Diplômé en 1958 de 1 'Ecole Polytechnique, option mines-géologie, 11 obtint en 1964 une mattrise en thermodynamique de l'Université de Birmingham.

M. GASTON POULIOT, est professeur titulaire au Département de Génie Minéral de l'Ecole Polytechnique de Montréal. Diplômé en géologie de l'Université de Montréal en 1956, 11 obtint une maftrise et un doctorat de l'Université McGill en 1958 et 1962. M. Pouliot commença sa carrière au service du Ministère des Richesses naturelles du Québec pour passer par la suite à la Commission géologique du canada en qualité de minéralogiste. 
M. ROLLAND VIAU, est conseiller pédagogique au Service pédagogique de l'Ecole Polytechnique depuis février 1978. Il détient les diplômes de B.Sc. Educ. et M.A. Educ. de I'Université de Montréal. M. Viau possède une importante expértence dans le domaine de 1 'enseignement.

M. FRED BASSAL, Ing., est professeur adjoint au Département de Génie Industriel de l'Ecole Polytechnique de Montréal. Diplômé en génie mécanique de l'Université du Calre en 1958, M. Bassal possède une vaste expêrience dans l'industrie à 1'échelle internationale. Il oeuvra au Conseil National de Recherche du Canada sur des projets de génie industriel dans les P.M.E. 